\title{
Reconstruction of cutaneous defects of the nasal tip and alar by two different methods
}

\author{
Yong Hun $\mathrm{Kim}^{1}$, \\ Hyung Woo Yoon ${ }^{1}$, \\ Seum Chung', \\ Yoon Kyu Chung ${ }^{1}$ \\ ${ }^{1}$ Department of Plastic and \\ Reconstructive Surgery, Wonju \\ Severance Christian Hospital, Yonsei \\ University Wonju College of Medicine, \\ Wonju; ${ }^{2}$ Department of Plastic and \\ Reconstructive Surgery, National Health \\ Insurance Corporation Ilsan Hospital, \\ Goyang, Korea
}

\begin{abstract}
Background: The alar and nasal tip are important subunits of the nose. Determining the optimal procedure for reconstructing a cutaneous defect in a nasal subunit depends on several factors including size, location, and involvement of deep underlying structures. We treated cutaneous defects after tumor ablation in the alar and nasal tip with a local flap, using an S-shaped design and a modified $\mathrm{V}-\mathrm{Y}$ advancement flap with a croissant shape.

Methods: We analyzed 36 patients with skin tumors who underwent flap coverage after tumor ablation. Rotation flaps were used in 26 cases and croissant-shaped $V-Y$ advancement flaps were used in 10 cases. The primary cause of the defects was skin cancer, except for one benign tumor. Results: The mean patient age was 71 years. The size of the defects ranged from $0.49 \mathrm{~cm}^{2}$ to 3.5 $\mathrm{cm}^{2}$. No recurrence of skin cancer was noted and all flaps lasted until the end of follow-up. Partial desquamation of the epidermis was noted in one case. The postoperative appearance for most patients was excellent, objectively and subjectively.

Conclusion: For cutaneous defects of up to about $4.0 \mathrm{~cm}^{2}$ of the alar and nasal tip, local flaps using our methods offered a good cosmetic and therapeutic result. The main advantage of our flaps is the minimal dissection required compared to bilobed and other local flap methods. We believe our flaps are a suitable option for alar and nasal tip reconstruction.
\end{abstract}

Keywords: Nose / Surgical flaps / Carcinoma / Basal cell

\section{INTRODUCTION}

Since the nose juts away from the face, it is more vulnerable to ultraviolet exposure and traumatic insult, especially in the nasal tip and alar area. Compared to the nasal dorsum, the skin of the nasal tip and alar area is thicker and tightly attached to the underlying alar cartilage. These characteristics make flap elevation around these areas more difficult. In addition, stronger sebaceous activity in these areas inevitably results in more scar formation than in any other part of the nose. As previously men-

\footnotetext{
Correspondence: Yoon Kyu Chung

Department of Plastic and Reconstructive Surgery, Wonju Severance Christian

Hospital, Yonsei University Wonju College of Medicine, 20 Ilsan-ro, Wonju 26426,

Korea

E-mail: ykchung@yonsei.ac.kr

Received October 27, 2018 / Revised November 26, 2018 / Accepted November 30, 2018
}

tioned, the nasal tip and alar are most commonly affected by skin cancers due to their prominence. Numerous surgical techniques for this part of the nose are well known. We performed cutaneous reconstruction for the nasal tip and alar areas with small to medium sized defects. Regardless of the chosen method, we attempted to minimize postoperative scars and deformities. When it came to reconstructing the nasal defect, the focus was on eradicating the underlying diseases, reserving normal tissue, and minimizing dissection of the adjacent tissue as much as possible. Skin grafts are a useful tool, but have some handicaps such as mismatched color and contour irregularity [1]. Although our methods are not new, we modified the flap design to simplify the rotation of the flap and incorporated a rotation advancement concept instead of simple advancement, as is typical in some cases of V-Y advancement flaps. By utilizing these 
modifications, we were able to obtain better objective and subjective results.

\section{METHODS}

We used two types of flaps: rotation flaps with an S-shape and a croissant-shaped modified V-Y flap. The rotation flap technique was used in 26 patients and the croissant-shaped modified V-Y flap was used in 10 patients. Patient ages ranged from 23 to 92 years old, with a mean age of 71 years (15 males and 21 females). Most of the defects were caused by tumor ablations: 32 for basal cell carcinoma, two for squamous cell carcinoma, one for a dermatofibroma, and one for a depressed scar from a previous skin graft after tumor excision. Wide excisions were performed in all cases except in two cases of scar revision (Tables 1,2). The resection margin was $0.2 \mathrm{~cm}$ and $0.3 \mathrm{~cm}$ for basal cell carcinoma and squamous carcinoma, respectively. The average size of the defect was $0.99 \mathrm{~cm}^{2}$ (Table 3). The alar area was operated on in 22 cases, the nasal tip in 12 cases. The rotation flap and croissant-shaped V-Y flap was designed after confirmation of clear resection margins on frozen sections. The rotation flap was designed to be performed in an adjacent area of the defect at a ratio of 1:1. The donation location was chosen based on skin laxity and expected contour deformity after flap replacement. The flap could have been based superomedially or superolaterally, though theoretically any base was possible. In case of the croissant-shaped modified V-Y flap, the long axis of the V-flap was 1.5-2 times the length of the diameter of the defect. The flap was elevated in the subcutaneous layer above the mimetic muscles. The bilateral tips of the horns of the V-flap were sutured to each other and fixed medially to cover the distal aspect of the defect [2]. The flap was then inset and fixed with sutures. Donor

Table 1. Patient characteristics

\begin{tabular}{|c|c|c|}
\hline Characteristics & Male & Female \\
\hline \multicolumn{3}{|l|}{ Patient } \\
\hline Number & 15 & 21 \\
\hline Age (mean, range) & 67 (23-92) & $74(53-87)$ \\
\hline \multicolumn{3}{|l|}{ Lesion } \\
\hline Basal cell carcinoma & 14 & 18 \\
\hline Squamous cell carcinoma & - & 2 \\
\hline Dermatofibroma & 1 & - \\
\hline Postoperative scar & - & 1 \\
\hline \multicolumn{3}{|l|}{ Location (subunit) } \\
\hline Alar & 11 & 13 \\
\hline Tip & 4 & 8 \\
\hline \multicolumn{3}{|l|}{ Defect size $\left(\mathrm{cm}^{2}\right)$} \\
\hline$<0.5$ & & 1 \\
\hline $0.51-1.00$ & & 29 \\
\hline $1.01-1.50$ & & 2 \\
\hline $1.51-2.0$ & & 2 \\
\hline$>2.0$ & & 2 \\
\hline
\end{tabular}

site closure was performed using the same method. A Penrose drain was inserted at the donor site in some cases.

\section{RESULTS}

The patients were 15 males (mean age, 67 years; range, 23 to 92 years) and 21 females (mean age, 74 years; range, 53 to 87 years). The reconstruction methods were rotation flap $(n=26)$ (Fig. 1) and croissant-shaped modified V-Y flap $(n=10)$ (Figs. 2, 3).

There were no complications involving hematoma, dehiscence, and flap necrosis except for one case of partial epidermal lysis. Stitches were removed 5 days postoperatively. Blunting of the upper portion of the nasolabial sulcus occurred in several cases when the alar defect was near the sulcus. In some cases, the flaps bulged, resulting in a lymphedema-like-appearance. No evidence of tumor recurrence was noted until the end of the follow-up period (7 months to 2 years; mean, 11 months). All patients showed satisfactory cosmetic outcomes.

\section{DISCUSSION}

Reconstruction of the alar and nasal tip remains a challenging task. The nose has eight specific subunits [3]. To reconstruct cutaneous defects of the nose, we must take each subunit into consideration. However, we have frequently encountered patients who require reconstructive surgery that violates multiple subunits of the nose. The skin of the alar and nasal tip is thicker and has more robust sebaceous activity but is less pliable than the remaining portions of the nose, which places constraints on a local flap construction. If a flap is forcibly constructed to cover a defect in this region, it results in distortion of the lower eyelid or alar without exception. Full thickness skin grafts seem to be an effective solution. Sometimes, the results can be satisfactory but frequently leave an unsightly scar, color discrimination, or depressed contour. Because of the similarity of the defect site, theoretically, a local flap adjacent to the defect would provide

Table 2. Distribution of nasal subunit and used flaps

\begin{tabular}{lcc}
\hline Variable & Rotation flap & Croissant flap $^{\text {a) }}$ \\
\hline Subunit & & \\
Alar & 17 & 7 \\
Tip & 9 & 3 \\
\hline
\end{tabular}

a)Croissant-shaped modified V-Y flap.

Table 3. Distribution of nasal subunit and defect area size $\left(\mathrm{cm}^{2}\right)$

\begin{tabular}{cccccc}
\hline Variable & $<0.5$ & $0.51-1.00$ & $1.01-1.50$ & $1.51-2.0$ & $>2.0$ \\
\hline Subunit & & & & & \\
Alar & 0 & 18 & 2 & 2 & 2 \\
Tip & 1 & 11 & 0 & 0 & 0 \\
\hline
\end{tabular}



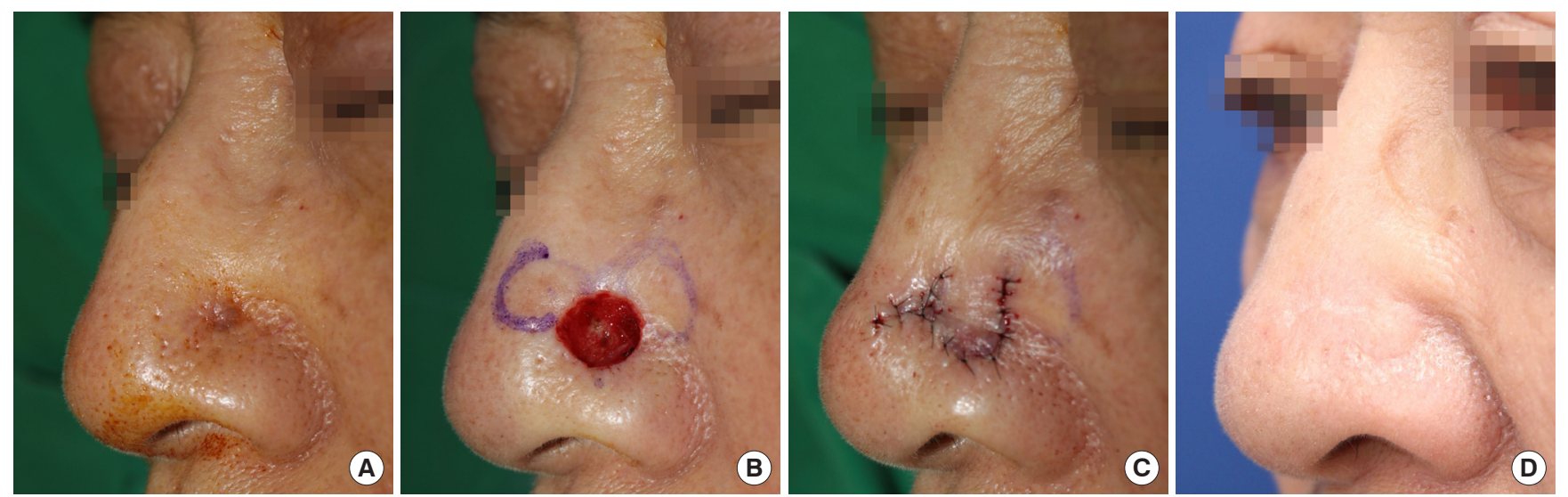

Fig. 1. A case of rotation flap on alar. (A) A 76-year-old woman with basal cell carcinoma of the left nasal alar. (B) After wide excision, defect size was $1 \mathrm{~cm} \times 1 \mathrm{~cm}$ and extended the full depth of the dermis. (C) The rotation flap was designed to be placed on the superomedial aspect of the defect. The defect was covered with the elevated flap. (D) Follow-up at 7 months after the procedure.
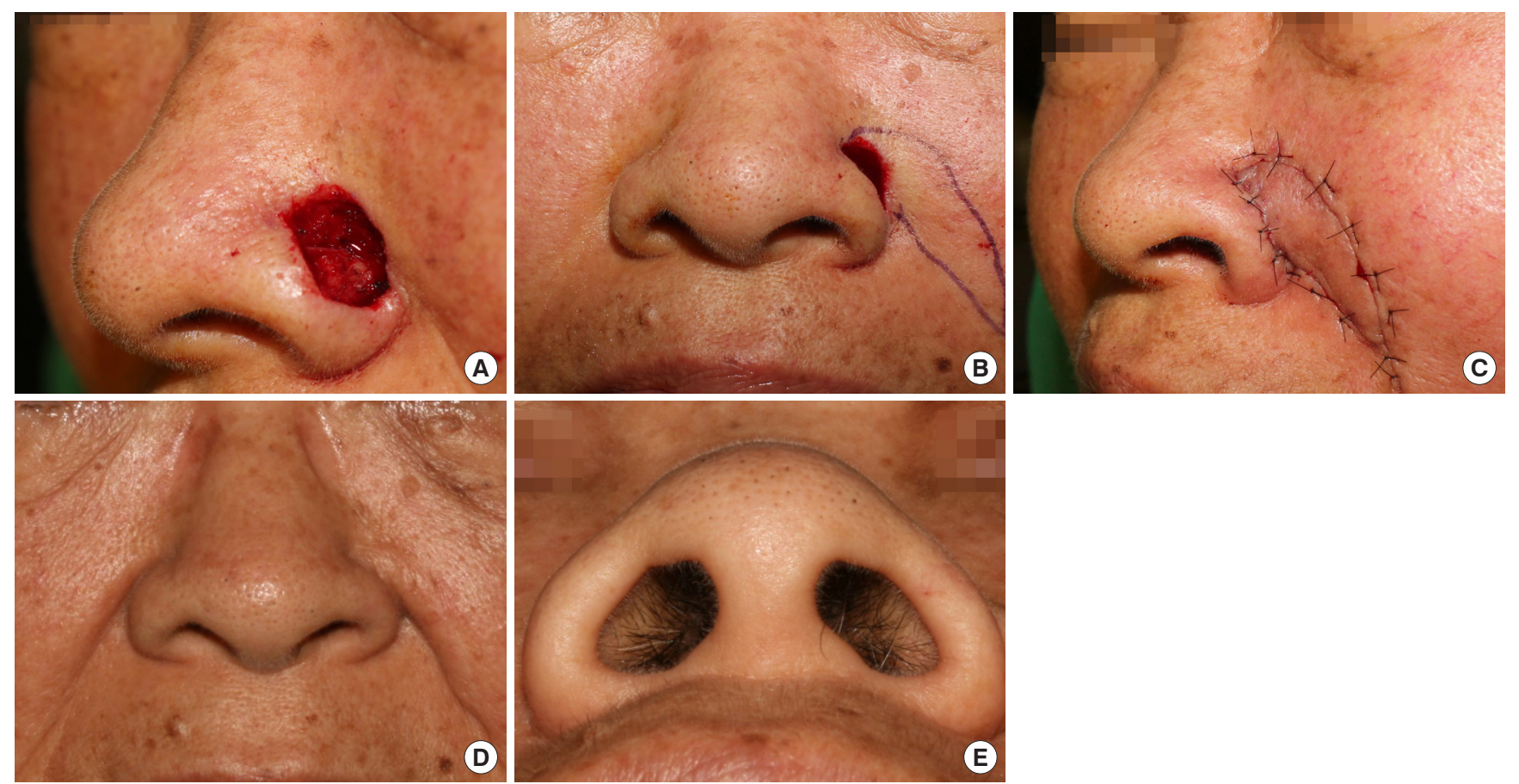

Fig. 2. A case of alar reconstruction. (A) A 73-year-old woman after wide excision of basal cell carcinoma on left alar. Resulting cutaneous defect size was $1.2 \mathrm{~cm} \times 1.0 \mathrm{~cm}$, the defect margin invaded the alar crease. (B) Considering the postoperative scar, the inferior margin of the flap was designed to lie along the nasolabial fold. (C) The defect was covered with croissant-shaped V-Y advancement flap. (D, E) Fifteen months later, the topographic appearance of the alar rim and the groove of the alar crease were well preserved with minimal postoperative scarring.

the best results. To improve the result cosmetically, various local flaps have been reported: bilobed flap [4,5], dorsal nasal flap, mesolabial flap [6], and a forehead flap [7,8]. All the flaps mentioned above provide excellent results and have their own advantages, but they require undermining of the adjacent tissues to achieve mobilization and may even require sacrifice of the remaining normal tissue. When reconstructing small and medium-sized defects around the nasal tip, those flaps are far from cost-efficient. The rotation flaps we used are designed to mini- mize dissection and decrease the subsequent contour distortion. We determined the flap size such that it was equal to that of the defect size, and by doing so, we made an effort to minimize resulting distortion of the nearby structures. In the croissant-shaped V-Y flap, the long axis of the V-flap was 1.5-2 times the length of the diameter of the defect [2]. Additionally, the procedure takes a short amount of time. All procedures were performed under local anesthesia. Most procedures took about 1 hour for the total operation time including confirma- 

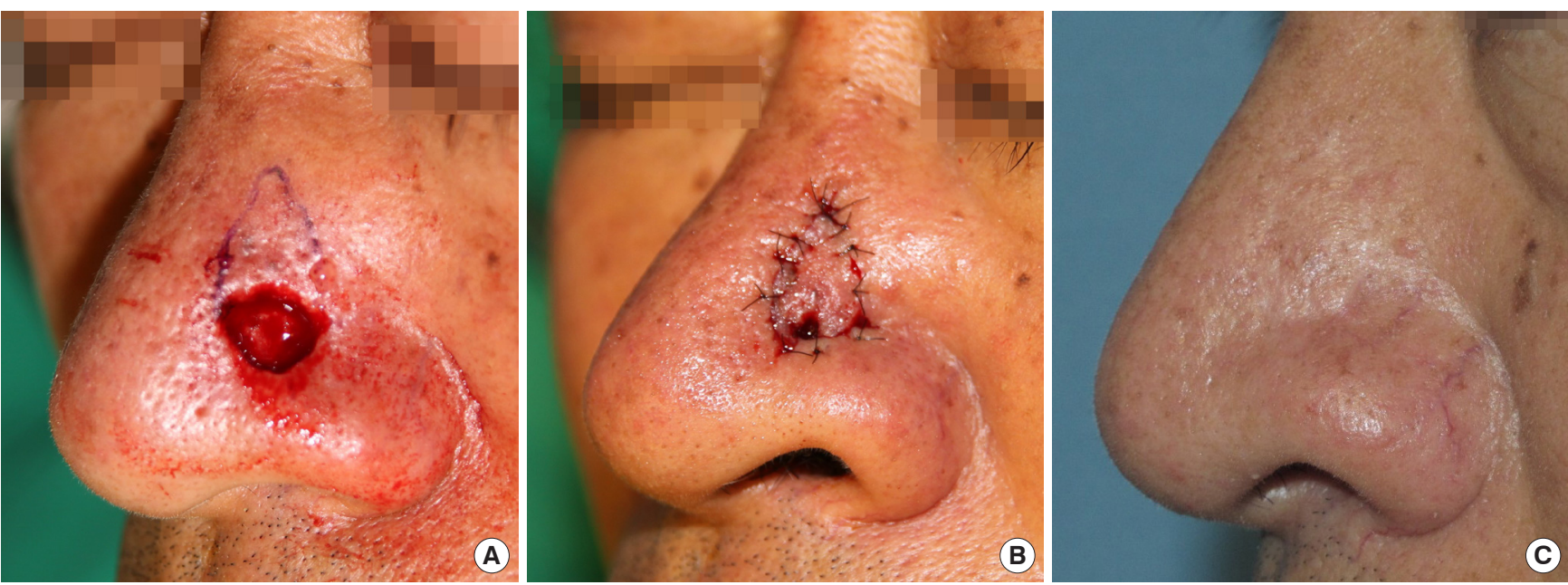

Fig. 3. Another method for alar reconstruction. (A) A 57-year-old man had a basal cell carcinoma on the nasal alar. About a $0.7 \mathrm{~cm} \times 0.7 \mathrm{~cm}$ sized defect resulted after resection of the tumor. (B) As the vicinity of the defect was near the relatively mobile side wall, a croissant-shaped $\mathrm{V}-\mathrm{Y}$ advancement flap was used. To prevent excessive tension, two lateral tips of the horn were not approximated but left open. The remaining small open wound healed with secondary intention. (C) A year later, an excellent outcome was noted without recurrence of the tumor.

tion of the frozen pathological sections. There were no major complications, such as total or partial flap necrosis, dehiscence, and infection. The topographic appearance of the nose can be altered with bilobed flap repair [7]. Nasal flaps have an inherent tendency to form a convex surface. This tendency can be further exacerbated by the development of a trapdoor deformity. A trapdoor scar developed in a few of our patients. To overcome this, a broader flap base can be designed. However, this may not be a significant problem for all patients. Sometimes, two adjacent subunits were invaded, but a significant contour distortion was not observed. This could be attributed to generous skin laxity as the majority of our patients were elderly. With our local flaps, we were able to achieve a meaningful aesthetic result in small to medium-sized defects of the nasal tip and alar areas.

\section{CONFLICT OF INTEREST}

YKC, a member of the Archives of Craniofacial Surgery, is the corresponding author of this article. However, he played no role whatsoever in the editorial evaluation of this article or the decision to publish it. Except for that, no potential conflict of interest relevant to this article was reported.

\section{PATIENT CONSENT}

The patients provided written informed consent for the publication and the use of their images.

\section{ORCID}

Yong Hun Kim https://orcid.org/0000-0002-6092-4374

Hyung Woo Yoon https://orcid.org/0000-0002-0216-1700

Seum Chung https://orcid.org/0000-0003-2963-881X

Yoon Kyu Chung https://orcid.org/0000-0002-0401-3912

\section{REFERENCES}

1. Lindsay KJ, Morton JD. Flap or graft: the best of both in nasal ala reconstruction. J Plast Reconstr Aesthet Surg 2015;68:13527.

2. Kim JY, Chung S, Chung YK. Croissant-shaped v-y advancement flap with 2 horns for repair of small- and medium-sized facial defects. J Craniofac Surg 2011;22:1781-4.

3. Burget GC, Menick FJ. The subunit principle in nasal reconstruction. Plast Reconstr Surg 1985;76:239-47.

4. Golcman R, Speranzini MB, Golcman B. The bilobed island flap in nasal ala reconstruction. Br J Plast Surg 1998;51:493-8.

5. Xue CY, Li L, Guo LL, Li JH, Xing X. The bilobed flap for reconstruction of distal nasal defect in Asians. Aesthetic Plast Surg 2009;33:600-4.

6. Yellin SA, Nugent A. Melolabial flaps for nasal reconstruction. Facial Plast Surg Clin North Am 2011;19:123-39.

7. Steiger JD. Bilobed flaps in nasal reconstruction. Facial Plast Surg Clin North Am 2011;19:107-11.

8. Correa BJ, Weathers WM, Wolfswinkel EM, Thornton JF. The forehead flap: the gold standard of nasal soft tissue reconstruction. Semin Plast Surg 2013;27:96-103. 